\title{
RECENT DEVELOPMENTS IN THE PROCEDURES USED AT THE SSCER LABORATORY FOR THE ROUTINE PREPARATION OF LITHIUM CARBIDE
}

\author{
VADIM V. SKRIPKIN and NIKOLAI N. KOVALIUKH
}

State Scientific Centre of Environmental Radiogeochemistry, Paladina 34, Kiev, 252680 Ukraine

\begin{abstract}
In this paper we describe and discuss the advantages from improvements in equipment design and operating procedures developed at the State Scientific Centre of Environmental Radiogeochemistry (SSCER) laboratory in Kiev. Two experimental areas are considered, viz.

1. The direct chemisorption into a lithium alloy of carbonaceous gases produced by the controlled thermal degradation (pyrolysis) of organic materials under vacuum. This approach offers the advantage of a single stage, highly efficient and economical procedure for the production of lithium carbide. It is applicable for most types of sample material encountered in routine dating work and including organic detritus dispersed in a highly (up to $95 \%$ by weight) mineral matrix and/or carbonates. Bone collagen can also be processed without the need for its prior extraction and purification.
\end{abstract}

2. A conical thin walled reaction vessel for achieving improved and reproducible recoveries in the production of lithium carbide from $\mathrm{CO}_{2}$ gas. This apparatus allows a much improved control over the surface dependent reaction in instances where there is no option other than the direct interaction of molten lithium with prepared $\mathrm{CO}_{2}$ gas.

\section{INTRODUCTION}

The measurement of natural radiocarbon concentrations by liquid scintillation counting (LSC) invariably depends on the production of lithium carbide as an intermediate in benzene synthesis (Barker 1953; Polach and Stipp 1967). In theory, the chemical reaction proceeds according to

$$
2 \mathrm{CO}_{2}+10 \mathrm{Li} \rightarrow \mathrm{Li}_{2} \mathrm{C}_{2}+4 \mathrm{LiO} .
$$

However, even if it can be achieved in practice this stoichiometric conversion has several disadvantages. In the first instance it is wasteful of lithium reagent since only $20 \%$ by weight is actually consumed in carbide production; the remainder is lost in combination with the oxygen of the $\mathrm{CO}_{2}$ molecules. The need to oxidize the sample carbon to $\mathrm{CO}_{2}$ as a preliminary stage is also time-consuming and requires a complex combustion system allied with cryogenic gas trapping facilities and serviced by a large-volume vacuum pumping capability. In attempts to offset these particular problems, several researchers (Starik, Arslanov and Klener 1963; Noakes, Kim and Stipp 1965; Arslanov and Tertychny 1970; Tamers 1975) developed and described procedures in which the raw sample material is first reduced to near elemental carbon by charring in an oxygen-free atmosphere and then reacted directly with lithium metal at $c a .800^{\circ} \mathrm{C}$,

$$
2 \mathrm{Li}+2 \mathrm{C} \rightarrow \mathrm{Li}_{2} \mathrm{C}_{2} \text {. }
$$

However, different practical problems are encountered with this approach. In particular for most sample types, the pre-charring (pyrolysis) stage invariably results in a significant loss of volatile organic distillates, which can amount to as much as $80 \%$ by weight of the original sample. It is therefore not suited in instances when only small samples are available, and it is also impractical when samples have a high admixture of inorganic (mineral) material. In such instances recourse to the preliminary production of $\mathrm{CO}_{2}$ gas and the conversion to carbide via Equation (1) is often unavoidable. Furthermore, when elemental carbon is allowed to react directly with molten lithium there is a sharp rise in temperature at the reaction surface, which if not adequately controlled, will result in the loss of reactants, which volatilize, condense and become unreactive on colder parts of the containment vessel. 


\section{Simultaneous Pyrolysis and Chemisorption}

Our answer to minimizing the loss of genuine sample carbon during pyrolysis has been via the development of a stainless steel reactor that allows thermal degradation of the raw sample to be carried out with simultaneous exposure of the volatile organics to a lithium melt. The system is designed to allow appropriate control of the required temperature differential between the discrete reaction zones. Two second-stage modifications to the experimental procedure are applicable for specific sample types and these allow the routine recovery of upwards of $95 \%$ of the raw sample carbon as high purity lithium carbide for subsequent hydrolysis to acetylene. The entire conversion procedure can be carried out in ca. $1 \mathrm{~h}$, and is effectively free of memory effects.

\section{Conical $\mathrm{CO}_{2} /$ Lithium Reaction Vessel}

As mentioned above, there are certain types of samples that leave no option other than to prepare lithium carbide by the direct injection of $\mathrm{CO}_{2}$ gas over a lithium melt. In such instances the conversion yield will be determined by maintaining optimum temperature control and ensuring that there is an adequate surface area of elemental lithium exposed to the residual gas pressure over the reaction surface. As mentioned previously, any inability to control areas of excessive temperature, due to the exothermic nature of the ongoing reaction, will result in losses caused by the condensation of reactants on the colder surfaces of the containment vessel. Equally important, however, is ensuring that unreacted $\mathrm{CO}_{2}$ has ready access to hot lithium metal; otherwise, competing reactions will result in the production of elemental carbon, viz.,

$$
2 \mathrm{Li}_{2} \mathrm{C}_{2}+\mathrm{CO}_{2} \rightarrow 2 \mathrm{Li}_{2} \mathrm{O}+5 \mathrm{C} .
$$

The occurrence of this and similar competing reactions is very evident by the presence of black particulate carbon in the lithium hydroxide waste solution remaining after subsequent hydrolysis of the carbide to produce acetylene. Such carbon losses are of course reflected in smaller amounts of benzene available for LSC. Perhaps even more important, however, is the fact that the incomplete conversion and recovery of sample carbon at any stage in the benzene synthesis procedure can give rise to a significant degree of isotopic fractionation, which has to be determined and factored into the calculation of accurate ${ }^{14} \mathrm{C}$ enrichment values. We have improved the conversion yield of lithium carbide (to routinely $>95 \%$ ) by designing a thin-walled conical reactor vessel that can be used to process up to $17.5 \mathrm{~L}$ of atmospheric $\mathrm{CO}_{2}(9.5 \mathrm{~g}$ carbon $)$ to carbide in $<50 \mathrm{~min}$ without excessive furnace heating.

\section{METHODS}

\section{Combined Pyrolysis and Chemisorption}

Figure 1 shows the design and construction details of the stainless steel reactor vessel employed to minimize the loss of sample carbon during pyrolysis. In use, the vessel is charged as shown with sample and lithium metal, sealed, pumped free of air and then introduced into a thermostatically controlled electric furnace. The temperature ranges designated for each reaction zone within the vessel are determined and maintained at the required differential by 1 ) fixing the optimum height of the titanium sample holder above the basal lithium melt, and 2) positioning the reactor vessel within the appropriate temperature gradient of the furnace jacket. The gaseous products from thermal decomposition of the sample are directed by its tubular holder onto the surface of the lithium. When the temperature regime is established correctly, the rate at which the volatile carbonaceous materials are absorbed chemically by the molten lithium is slightly greater than that at which they are being produced in the pyrolysis zone. Under these conditions, the residual gas pressure within the reactor is maintained in the range 0.1 to 0.2 atmospheres, which favors an increase in the yield of molecularly light products from the thermal dissociation and also prevents condensation of these volatiles onto the 
reactor walls. There is a high efficiency in lithium carbide production and with some parallel formation of oxide, hydride and nitride salts from the occurrence of the corresponding chemical elements in the organic condensates. Completion of the simultaneous pyrolysis/chemisorption reactions is noticed by a drop in residual pressure below 0.05 atmospheres when the temperature in the pyrolysis zone has attained its maximum value $\left(600-650^{\circ} \mathrm{C}\right)$. The reactor is then pumped for a short time to ensure the decomposition of any lithium hydride and/or lithium nitride produced by chemisorption. The vessel is then allowed to cool to room temperature under sealed vacuum. For typical samples in the size range 5 to $15 \mathrm{~g}$ carbon, this stage in the overall carbide production sequence takes $<40 \mathrm{~min}$, including the time required for heating and cooling of the reactor vessel. While up to $80 \%$ of the available sample carbon may be volatilized and converted to lithium carbide during the simultaneous pyrolysis/chemisorption reactions, there is invariably a solid carbonaceous residue comprising charcoal and/or other thermally stable organic species. To ensure that such carbon is also converted to lithium carbide we employ one of two second-stage modifications, depending on the sample type.

Where the initial sample was largely organic in composition and with low concentrations of phosphorous, sulphur and other inorganic compounds, e.g., peat or wood, the "charcoal" residues are simply transferred to the basal zone in the reactor. The vessel is resealed, pumped free of air at room temperature and then heated again to $800^{\circ} \mathrm{C}$. This completes the conversion of residual carbon to carbide.

A second adaptation is applied for samples that have a relatively high mineral content or comprise demineralized bone protein. In such instances, an excess of manganese dioxide is added to the charred residue which is retained within the pyrolysis reaction zone. The reactor vessel is resealed, pumped free of air and subjected to a second cycle of differential heating. The manganese dioxide begins to decompose, releasing free oxygen at temperatures above $530^{\circ} \mathrm{C}$ and this favors the predominant formation of $\mathrm{CO}$, which reacts with the lithium melt in the base of the reactor vessel,

$$
4 \mathrm{Li}+2 \mathrm{CO} \rightarrow \mathrm{Li}_{2} \mathrm{C}_{2}+2 \mathrm{LiO} .
$$

The dual reaction zone vessel is also used for processing carbonate samples via a single-cycle heating process. Calcium carbonate dissociates $\left(\mathrm{CaCO}_{3} \rightarrow \mathrm{CaO}+\mathrm{CO}_{2}\right)$ on heating to give a partial pressure of 0.1 to 0.2 atmospheres $\mathrm{CO}_{2}$ between 500 to $560^{\circ} \mathrm{C}$. The upper temperature zone is set to progress this evolution of chemically pure $\mathrm{CO}_{2}$, which is simultaneously absorbed into the basal melt of lithium held at $750-800^{\circ} \mathrm{C}$. The stoichiometric requirement for excess lithium (as in Equation 1) prevails but calcium based impurities do not interfere with the yield or purity of the resultant carbide.

\section{The Conical Reaction Vessel}

The design of the alternative thin-walled conical reaction vessel that we use to produce lithium carbide from pre-prepared $\mathrm{CO}_{2}$ gas (Equation 1) is shown in Figure 2. Pertinent features of the configuration and construction are:

1. The relatively large $\left(100 \mathrm{~cm}^{2}\right.$ base $)$ to maximize the surface area available for direct interaction of $\mathrm{CO}_{2}$ and lithium.

2. The thin walls (2-mm gauge stainless steel) which facilitate rapid heat exchange and precise temperature control via an inexpensive low-power heater plate (800-watt domestic stove element).

3. The $c a .1 \mathrm{~L}$ internal volume, which is adequate for the processing of up to $17.5 \mathrm{~L}$ atmospheres of $\mathrm{CO}_{2}(9.5 \mathrm{~g}$ carbon).

The experimental conditions of temperature and gas pressure used to initiate and control the progress of lithium carbide formation are as described by Arslanov et al. (1993). No memory effects are encountered between sample runs and completion of the process requires $<50 \mathrm{~min}$. 


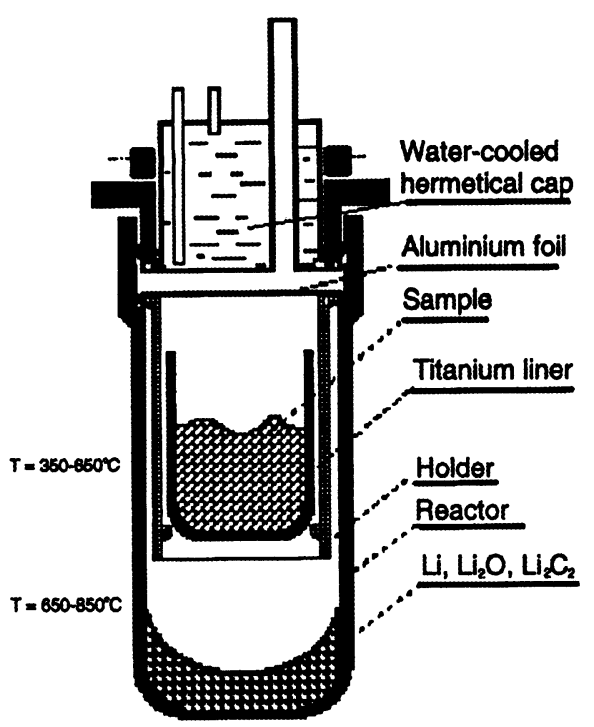

Fig. 1. Stainless steel reactor vessel for lithium carbide production

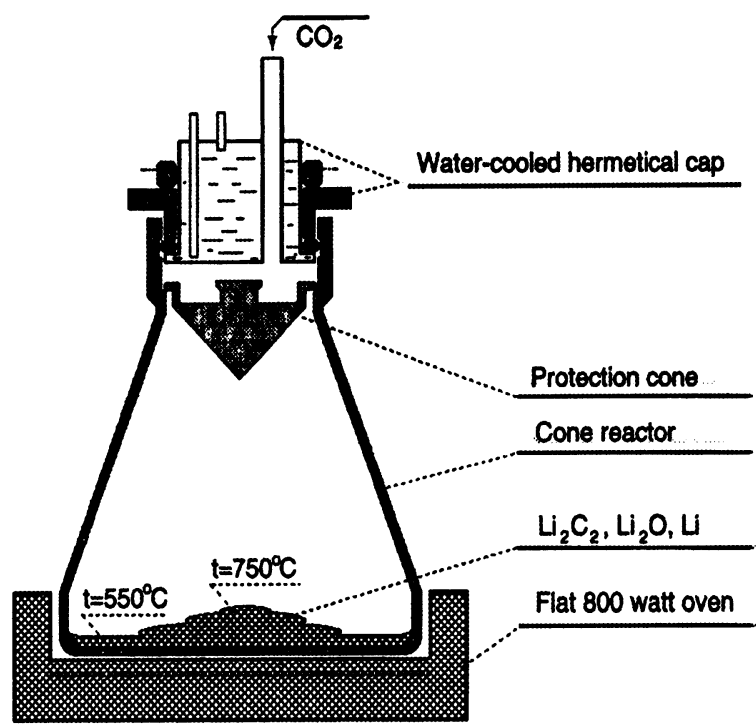

Fig. 2. Thin-walled conical reaction vessel for lithium carbide production

\section{CONCLUSION}

The equipment and procedures described here have been of considerable overall advantage in our ongoing development of an economically viable program of natural ${ }^{14} \mathrm{C}$ measurements. They represent a significant saving in time (manpower) and costs (of both chemical reagents and vacuum equipment). They have proven to be appropriate for analyses of a wide range of sample types and routinely produce high $(>95 \%$ ) yields in the conversion of sample carbon to high-purity lithium carbide. The combined vacuum pyrolysis/chemisorption procedure is particularly advantageous where small samples have to be processed to benzene for LSC.

\section{ACKNOWLEDGMENT}

We thank D. D. Harkness for improving the English text and offering many valuable suggestions during the preparation of this article.

\section{REFERENCES}

Arslanov, Kh. A. and Tertchny, N. I. 1970 On acetylene synthesis from $\mathrm{CO}_{2}$ for radiocarbon dating. In Astrophysical Phenomena and Radiocarbon. Tbilisi University: $55-60$.

Barker, H. 1953 Radiocarbon dating: Large-scale preparation of acetylene from organic material. Nature 172: 631-632.

Noakes, J. E., Kim, S. M. and Stipp, J. J. 1965 Chemical and counting advances in liquid scintillation age dating. In Radiocarbon and Tritium Dating: Proceedings of the 6th International Conference on Radiocarbon and Tritum Dating, Washington State University, Pullman, Wash., June 7-11, 1965. Oak Ridge, Tennessee,
U.S. Atomic Energy Commission: 650-652.

Polach, H. A. and Stipp, J. J. 1967 Improved synthesis techniques for methane and benzene radiocarbon dating. International Journal of Applied Radiation and Isotopes 18: 359-364.

Starik, I. E., Arslanov, Kh. A. and Klener, I. R. 1963 Improved chemical procedure of sample treatment for radiocarbon dating by means of scintillation technique. Radiokhimiya 5(2): 198-205.

Tamers, M. A. 1975 Chemical yield optimisation of the benzene synthesis for radiocarbon dating. International Journal of Applied Radiation and Isotopes 26(11): 676-683. 